Renata L. Riha

Cite as: Riha RL. Oxygen for the treatment of obstructive sleep apnoea hypopnoea syndrome. Breathe 2019; 15: e104-e107.

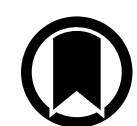

CrossMark

(C)ERS 2019

\title{
Editorial
}

\section{Oxygen for the treatment of obstructive sleep apnoea hypopnoea syndrome}

Oxygen, in the form of diatomic oxygen $\left(\mathrm{O}_{2}\right)$, comprises $20.8 \%$ of the Earth's atmosphere and is essential to most life forms on the planet. In 1777, Lavoisier was the first to name oxygen and recognise that it was a chemical element involved in combustion.

Supplemental oxygen for the treatment of human pathophysiological states has been around for over 100 years, applied at hyperbaric, hypobaric and sea level pressures. It is a life-saving treatment when there is insufficient inspired oxygen acutely; however, there is increasing recognition that hyperoxaemia (resulting from administration of the gas at supra-normal partial pressures) can be harmful if given for prolonged periods of time or in inappropriate situations (e.g. premature babies). The liberal use of supplemental oxygen in acutely ill patients without hypoxaemia is associated with a significantly increased relative risk of death at 30 days after hospitalisation [1, 2]. In 2015, the AVOID trial demonstrated that supplemental oxygen $\left(8 \mathrm{~L} \cdot \mathrm{min}^{-1}\right)$ in normoxic patients with ST-elevation myocardial infarction was detrimental, resulting in increased early myocardial injury and larger myocardial infarct size at 6 months, measured using cardiac magnetic resonance imaging [3].

By contrast, in conditions characterised by hypoxaemia, the introduction of low-flow oxygen has been shown to prolong life acutely and chronically. In the classic example from respiratory medicine, quality of life and survival have been shown to improve with long-term oxygen therapy in COPD patients; but these patients have chronic, rather than intermittent hypoxaemia, $24 \mathrm{~h}$ a day, in addition to hypercapnia [4, 5].

\section{Trials of nocturnal oxygen therapy in obstructive sleep apnoea}

Obstructive sleep apnoea (OSA) is characterised by repetitive episodes of upper airway collapse, resulting in a fall in oxygen saturation and a rise in carbon dioxide at the end of each apnoea. A large proportion of the population suffers from OSA (up to $20 \%$ in industrialised countries) and 5-7\% suffer from the syndrome which is characterised by excessive daytime somnolence and impairment in mood, memory and concentration [6]. Currently, the "gold standard" for treating moderate-to-severe OSA is continuous positive airway pressure (CPAP), which abolishes apnoeas and hypopnoeas and with them the intermittent hypoxia and arousals that are the mechanisms leading to long-term cardiovascular and metabolic morbidities [6]. However, CPAP comes with a low, long-term adherence rate of $40-60 \%$ [7].

The attraction of using long-term inspired oxygen therapy to correct the intermittent nocturnal hypoxaemia, in preference to a cumbersome interface attached to an air compressor, is thus evident. 
In 2013, MEHTA et al. [8] published a literature review of all 14 studies published up until 2011 that fulfilled the following criteria: 1) the studies were prospective trials, 2) the target population had OSA, 3) the study intervention was oxygen therapy and/or CPAP therapy, and 4) the variables studied included the effects of oxygen on the apnoea-hypopnoea index (AHI), nocturnal hypoxaemia and apnoea duration. The studies included a total of 359 patients; nine studies were cohort studies and five were randomised controlled studies with three intervention arms: oxygen alone, CPAP and placebo/sham-CPAP. The study duration in all studies ranged from one night to 3 months, $89 \%$ of all subjects were male, and overall, most patients had moderate-to-severe OSA with an $\mathrm{AHI}$ ranging from $20.5 \pm 5$ to $88.2 \pm 27$ events $\cdot \mathrm{h}^{-1}$. In the published randomised controlled trials of oxygen versus CPAP versus placebo/sham-CPAP (duration ranging from 2 weeks to 3 months), nocturnal oxygen raised the mean lowest oxygen saturation measured by pulse oximetry $\left(\mathrm{S}_{\mathrm{pO}_{2}}\right)$ statistically significantly higher versus the other interventions. The effects on all other parameters, including the Epworth Sleepiness Score (ESS), blood pressure and $\mathrm{AHI}$ were variable and not statistically significant overall. Eight cohort studies, which examined the effects of supplemental oxygen versus air alone, but not CPAP, revealed variable reductions in $\mathrm{S}_{2}$ recorded overnight, with three studies documenting prolongation of apnoeas and hypopnoeas on the study night in those using oxygen. Overall, CPAP and nocturnal administration of oxygen both resulted in equivalent improvements in $\mathrm{S}_{\mathrm{PO}_{2}}$, but only CPAP lowered the $\mathrm{AHI}$, blood pressure parameters and ESS. In all the studies, nocturnal oxygen was delivered at a rate of 2-3 L· $\mathrm{min}^{-1}$.

In 2014, Gottlieb et al. [9] published the results of the Heart Biomarker Evaluation in Apnea Treatment (HeartBEAT) study. This randomised, parallel-group, controlled trial which enrolled a final analysis cohort of 281 patients with OSA, aimed to assess the effects of CPAP versus nocturnal supplemental oxygen versus lifestyle education only in reducing cardiovascular risk.

Patients aged $45-75$ years were recruited from across four cardiology practices and randomised to one of the three treatment arms. The majority of patients were men ( $>75 \%$ of the total group) and patients were identified as having OSA on the basis of a limited study performed in an unattended setting using a Type III device. Randomisation into the trial was offered to those with an apnoeas and hypopnoeas per hour in bed $(A+H)=15$ events $\cdot h^{-1}$ (incorrectly reported as an $\mathrm{AHI}$ ). Those with an $\mathrm{A}+\mathrm{H}>50$ events $\cdot \mathrm{h}^{-1}$ or an oxygen desaturation $<85 \%$ for more than $10 \%$ of the recording were excluded from the study, in addition to anyone with a central apnoea index over 5 events $\cdot \mathrm{h}^{-1}$. Median time spent with $\mathrm{SpO}_{2}<90 \%$ ranged from
$3.8 \%$ to $6 \%$ of the recording time across the groups. Nocturnal supplemental oxygen was delivered via nasal prongs at $2 \mathrm{~L} \cdot \mathrm{min}^{-1}$ and mean \pm sD duration of use was $4.8 \pm 2.4 \mathrm{~h}$ per night, significantly greater than the $3.5 \pm 2.7 \mathrm{~h}$ per night duration of CPAP use $(p=0.001)$. There was no significant effect of nocturnal oxygen on blood pressure parameters at 3 months in comparison to CPAP.

By contrast, a more recent publication by TURNBULL et al. [10], using the 2-week CPAP withdrawal method found the opposite results. In this randomised, double-blind, cross-over trial with final analysis of 25 patients, supplemental oxygen at $5 \mathrm{~L} \cdot \mathrm{min}^{-1}$ via a mask was shown to significantly attenuate rebound rise in systolic and diastolic blood pressures and median oxygen desaturation index (ODI) after CPAP withdrawal. Blood pressure was measured in the morning, or late morning. Whether the patients were supine or not was not documented. Despite reducing intermittent hypoxia, supplemental oxygen had a minimal effect on $\mathrm{AHI}$, heart rate, urinary markers of sympathetic activation, or objective and subjective sleepiness. Average use of the supplemental oxygen was $7.2 \mathrm{~h}$ a night. Patients appeared to have more severe OSA on inclusion in the trial compared with the HeartBEAT study [9], with a median (interquartile range) $>4 \%$ ODI of $48(25.3-68.2)$ events $\cdot h^{-1}$ at baseline diagnosis.

\section{High-flow nasal cannula in the treatment of OSA}

High-flow nasal cannula (HFNC) can be useful for delivering heated and humidified medical gas flows up to $60 \mathrm{~L} \cdot \mathrm{min}^{-1}$, even in an open system (mouth and nasal breathing combined) [11]. HFNC delivers oxygen using an air/oxygen blender with an inspiratory oxygen fraction $\left(\mathrm{F}_{\mathrm{IO}_{2}}\right)$ set between 0.21 and 1 , at a rate of up to $60 \mathrm{~L} \cdot \mathrm{min}^{-1}[11]$. HFNC has other physiological advantages over different oxygen delivery systems in that it is thought to reduce dead space, provide a constant $\mathrm{FIO}_{2}$ and decrease positive end-expiratory pressure. In the case of OSA, it is thought to be less intrusive than CPAP and reduces upper airway obstruction by increasing pharyngeal pressure as well as reflexively reducing ventilatory drive [12]. HFNC was first trialled and reported in adults in 2007 [12], and in children in 2009 [13]. Subsequently, a study was undertaken to assess its effectiveness in adults with acute stroke [14] and in a small trial of CPAP intolerant children [15]. None of these studies have used anything other than air $\left(\mathrm{FIO}_{2}, 0.21\right)$, so no comment can be made about oxygen enrichment in the management of these patients' OSA. Perhaps a study using HFNC with different $\mathrm{FIO}_{2}$ settings is a future possibility, in view of the considerations below. 


\section{Phenotyping OSA}

In recent years, there has been an upsurge in phenotyping patients with obstructive sleep apnoea hypopnoea syndrome (OSAHS) in an effort to improve adherence to tailored therapies (for examples see [16, 17]). With our increased understanding of pathophysiological mechanisms resulting in nocturnal upper airways obstruction, endotypes are also being identified. In response to supplemental oxygen therapy, loop gain is known to fall in patients with OSA, and in 2014, EDWARDs et al. [18] assessed the effects of hypoxia and hyperoxia on four physiological traits in 11 patients with OSA: pharyngeal collapsibility, loop gain, upper airway responsiveness and arousal threshold. Hyperoxia $\left(\mathrm{F}_{\mathrm{IO}_{2}}\right.$ 0.5) lowered loop gain; while hypoxia $\left(\mathrm{FIO}_{2} \sim 0.15\right)$ increased loop gain, increased arousal threshold and improved pharyngeal collapsibility [18]. This was followed by a trial to determine whether eszopiclone and nocturnal oxygen therapy could improve $\mathrm{AHI}$ in 20 randomly recruited subjects [19]. Those with the least upper airway collapsibility were most likely to respond with a reduction, but not abolition, of the $\mathrm{AHI}$ and improvement in intermittent hypoxaemia. In order to aid clinical decision making in choosing this therapy in preference to CPAP, the authors went on to retrospectively assess whether a patient's therapeutic CPAP requirement could act as a predictor of responsiveness to eszopiclone and oxygen; a CPAP level of $<8 \mathrm{cmH}_{2} \mathrm{O}$ resulted in $78 \%$ sensitivity and $82 \%$ specificity indicating reduced upper airway collapsibility [20]. More recently, the same group have examined markers on polysomnography to identify OSA responders to supplemental nocturnal oxygen at an $\mathrm{FlO}_{2}$ of 0.4 [21].

\section{A role for nocturnal oxygen therapy in OSA?}

To date, all the studies exploring this question have variable baselines of hypoxia and normoxia, focus on variable outcome measures and have delivered variable amounts of supplemental oxygen for a maximum duration of 3 months. An OSA patient with intermittent hypoxia experiencing falls in oxygen from $94 \%$ to $85 \%$ will be very different to a patient whose apnoeas/hypopnoeas are scored on the basis of a fall in $\mathrm{SpO}_{2}$ from $95 \%$ to $91 \%$. Trials aimed at identifying phenotypes and endotypes of OSAHS have focused on small numbers of subjects recruited from among thousands of patients with this condition. The majority of studies have deployed 2-3 L. $\mathrm{min}^{-1}$ using nasal prongs, apart from the study by TURNBULL et al. [10] which used $5 \mathrm{~L} \cdot \mathrm{min}^{-1}$ via mask overnight.

The overwhelming level of evidence currently mitigates against the routine use of nocturnal oxygen therapy in OSA patients, not least because the long-term consequences of chronic nocturnal administration of oxygen are unknown at either 2 or $5 \mathrm{~L} \cdot \mathrm{min}^{-1}$ in those whose baseline $\mathrm{SpO}_{2}$ may not be in the hypoxic range to begin with. Suppression of hypoxic respiratory drive with concomitant rises in hypercarbia and acidosis has also not been assessed fully in these patient cohorts, even without concomitant respiratory pathology. Perhaps in the future, with better, more flexible and easier methods of clinically identifying endotypes of OSA, oxygen therapy alone, or in combination with other therapies may be justifiable. At present, we are still a long way from this approach.

\section{Affiliations \\ Renata L. Riha \\ Dept of Sleep Medicine, Royal Infirmary of Edinburgh, Edinburgh, UK.}

\section{Conflict of interest}

R.L. Riha has nothing to disclose.

\section{References}

1. Chu DK, Kim LH, Young PJ, et al. Mortality and morbidity in acutely ill adults treated with liberal versus conservative oxygen therapy (IOTA): A systematic review and meta-analysis. Lancet 2018; 391: 1693-1705.

2. McEvoy JW. Excess oxygen in acute illness: adding fuel to the fire. Lancet 2018; 391: 1640-1642.

3. Stub D, Smith K, Bernard S, et al. Air Versus Oxygen in ST-Segment-Elevation Myocardial Infarction. Circulation 2015; 131: 2143-2150.

4. Continuous or nocturnal oxygen therapy in hypoxemic chronic obstructive lung disease: a clinical trial Nocturnal Oxygen Therapy Trial Group. Ann Intern Med 1980; 93 391-398.
5. Long term domiciliary oxygen therapy in chronic hypoxic cor pulmonale complicating chronic bronchitis and emphysema Report of the Medical Research Council Working Party. Lancet 1981; 1: 681-686.

6. Jennum P, Riha RL. Epidemiology of sleep apnoea/hypopnoea syndrome and sleep-disordered breathing. Eur Respir J 2009; 33: 907-914.

7. Bakker JP, Weaver TE, Parthasarathy S, et al. Adherence to CPAP: what should we be aiming for, and how can we get there? Chest 2019; 155: 1272-1287.

8. Mehta V, Vasu TS, Phillips B, et al. Obstructive sleep apnea and oxygen therapy: a systematic review of the literature and meta-analysis. J Clin Sleep Med 2013; 9: 271-279. 
9. Gottlieb DJ, Punjabi NM, Mehra R, et al. CPAP versus oxygen in obstructive sleep apnea. N EnglJ Med 2014; 370: 2276-2285.

10. Turnbull CD, Sen D, Kohler M, et al. Effect of supplemental oxygen on blood pressure in obstructive sleep apnea (SOX). A randomized continuous positive airway pressure withdrawal trial. Am J Respir Crit Care Med 2019; 199: 211-219.

11. Nishimura M. High-flow nasal cannula oxygen therapy in adults: physiological benefits, indication, clinical benefits, and adverse effects. Respir Care 2016; 61: 529-541.

12. McGinley BM, Patil SP, Kirkness JP, et al. A nasal cannula can be used to treat obstructive sleep apnea. Am J Respir Crit Care Med 2007; 176: 194-200.

13. McGinley B, Halbower A, Schwartz AR, et al. Effect of a high-flow open nasal cannula system on obstructive sleep apnea in children. Pediatrics 2009; 124: 179-188.

14. Haba-Rubio J, Andries D, Rey V, et al. Effect of transnasal insufflation on sleep disordered breathing in acute stroke: a preliminary study. Sleep Breath 2012; 16: 759-764.

15. Hawkins S, Huston S, Campbell K, et al. High-flow, heated, humidified air via nasal cannula treats CPAP-intolerant children with obstructive sleep apnea. J Clin Sleep Med 2017 13: 981-989.
16. Zinchuk AV, Gentry MJ, Concato J, et al. Phenotypes in obstructive sleep apnea: A definition, examples and evolution of approaches. Sleep Med Rev 2017; 35: 113-123.

17. Eckert DJ. Phenotypic approaches to obstructive sleep apnoea - New pathways for targeted therapy. Sleep Med Rev 2018; 37: 45-59.

18. Edwards BA, Sands SA, Owens RL, et al. Effects of hyperoxia and hypoxia on the physiological traits responsible for obstructive sleep apnoea. J Physiol 2014; 592: 4523-4535.

19. Landry SA, Joosten SA, Sands SA, et al. Response to a combination of oxygen and a hypnotic as treatment for obstructive sleep apnoea is predicted by a patient's therapeutic CPAP requirement. Respirology 2017; 22: 1219-1224.

20. Edwards BA, Sands SA, Owens RL, et al. The combination of supplemental oxygen and a hypnotic markedly improves obstructive sleep apnea in patients with a mild to moderate upper airway collapsibility. Sleep 2016; 39: 1973-1983.

21. Sands SA, Edwards BA, Terrill PI, et al. Identifying obstructive sleep apnoea patients responsive to supplemental oxygen therapy. Eur RespirJ 2018; 52: 1800674 